\title{
Pesticides induced oxidative stress and histomorphological changes in liver and kidney of female Bandicota bengalensis and Tatera indica
}

\author{
Shasta Kalra and Gurinder Kaur Sangha* \\ Department of Zoology, Punjab Agricultural University, Ludhiana-141004 (Punjab), INDIA \\ *Corresponding author. E-mail: shastakalra@yahoo.com \\ Received: September 7, 2016; Revised received: January 22, 2017; Accepted: April 30, 2017
}

\begin{abstract}
The liver and kidney of female Bandicota bengalensis and Tatera indica rats collected from Bathinda region of south west region of Punjab were used to investigate the toxic effects of these pesticides. Levels of total proteins decreased in all the rats collected from study area. Activity levels of different OS parameters: catalase (CAT), superoxide dismutase (SOD), glutathione transferase (GST), glutathione reductase (GR) and glutathione peroxidise (GPx) were differentially altered and the product of oxidation namely, malondialdehyde increased in liver $(11.05 \pm 0.18,9.02 \pm 0.26$ in control rats increased to $11.60 \pm 0.10,9.43 \pm 2.58$ in study area $B$. bengalensis and $T$. indica rats respectively) and kidney $(10.03 \pm 0.44,8.68 \pm 0.24$ in control rats increased to $10.18 \pm 0.72,8.92 \pm 4.34$ in study area $B$. bengalensis and $T$. indica rats respectively) of rats collected from study area as compared to control rats. Histomorphological studies further revealed number of abnormalities as infiltration, vacuolization, enlarged sinusoids and necrosis in liver of Bathinda rats, while renal histo architecture of kidney showed high degeneration of glomeruli. The results infer that environmental contaminants mainly pesticides leads to number of pathophysiological conditions in the liver and kidney and for altering antioxidant defence system in rats inhabiting south- west region of Punjab.
\end{abstract}

Keywords: Antioxidants, Environmental contaminants, Oxidative stress, Pesticides, Proteins

\section{INTRODUCTION}

Pesticides, though present in the environment in small quantities as compared to other contaminants such as industrial wastes and fertilizers may causes toxicity through direct damage to cells, interference with biochemical processes necessary for normal cell function and biotransformation resulting in toxic metabolites (Hashmi and Khan, 2011). These have been shown to induce the production of reactive oxygen species (ROS) which ultimately lead to a condition of oxidative stress (OS) (Abdollahi et al., 2004). Pesticides induce OS through ROS production has been evidenced by the increased level of malondialdehyde (MDA) i.e. end product of lipid peroxidation (LPO) and by differentially modified endogenous antioxidants like catalase (CAT), glutathione-S-transferase (GST), superoxide dismutase (SOD) and glutathione peroxidase (GPx) which can lead to the development of OS in different tissues having moderate to severe pathophysiological consequences (Sharma and Sangha, 2014).

The Malwa region of Punjab, India, is less than $15 \%$ of the total area of Punjab (only $0.5 \%$ of the total geographical area of India), but it consumes nearly $75 \%$ of the total pesticides used in Punjab. The high use of pesticides, along with environmental and social factors, is responsible for the high concentration of pesticide residues in the food chain of this region
(Mittal et al., 2013). Agriculture is the major occupation of the South West region's people of Punjab (Thakur et al., 2008). Cotton is a major crop in the Malwa region, which requires more use of pesticides to control the infestation of pests, as the pests have developed resistance to the pesticides (Shetty, 2004). The farmers and primarily agricultural workers are inadvertently exposed to pesticides through occupational use at all stages starting from pesticide purchase, transport, storage, dilution of pesticide concentrate, leaking of spray equipment, and inhalation during pesticide spraying (Maroni et al., 2000).

Liver and kidney are the most sensitive organ against pesticide toxicity and damage, as they play key role in the metabolism and elimination of pesticides (Sharma and Sangha, 2014). The susceptibility of these tissues to the stress due to exposure to pesticides is a function of the overall balance between the degree of OS and antioxidant activity (Mansour and Mossa, 2010). Earlier studies have revealed significant and progressive degenerative changes in liver of pesticide treated rats (Sharma and Sangha, 2014). As kidney is central metabolic organ, along with liver, important for metabolic and excretory processes, so the present study was designed to investigate the toxic effects of pesticides in terms of oxidative stress biomarkers and on histopathological features of liver and kidney of female adult albino rats. 


\section{MATERIALS AND METHODS}

Chemicals: All the chemicals were purchased from Sigma-Aldrich, SDFCL (SD Fine-Chem Ltd) and SRL (Sissco Research Laboratories Pvt. Ltd). All chemicals used were either of analytical grade or the highest purity commercially available.

Animals: Female field rats Bandicota bengalensis and Tatera indica were trapped from fields of Bathinda district of South West region of Punjab. Same species of rats were also collected from PAU, Ludhiana and adjoining areas and they served as control rats. All methods and procedures of animal handling during research were conducted in accordance with the guidelines of Committee for the Purpose of Control and Supervision of Experiments on Animals (CPCSEA), India and experiments conducted in the present study were duly approved by Institutional Animal Ethics Committee (IAEC), Guru Angad Dev Veterinary and Animal Sciences University, Ludhiana vide letter no 3901-35 dated 06-08-2012.

Experimental design: Animals were brought to laboratory, separated according to species and observed for morphological symptoms.

Organ weight and Organosomatic index: Following humane sacrifice, the vital organs viz liver and kidney were excised, cleaned off the adhering tissue and weighed separately. The organo-somatic index (OSI) was calculated by using the following formula as per Chattopadhyay et al. (2011).

Organo - somatic Index $($ OSI) $=$ Organ weight (g) / Live body weight (g) X 100

Sample preparation : For biochemical studies, $0.5 \mathrm{~g}$ of liver and one whole kidney was homogenized in 2 $\mathrm{ml}$ of phosphate buffer saline (PBS 0.1M, pH 7.4) and the homogenate was centrifuged at 3000 for r.p.m. for $10 \mathrm{~min}$. Supernatant was used for estimation of total proteins by method of Lowry et al., (1951), CAT (catalase) by Aebi, (1983), SOD (Superoxide Dismutase) by Marklund and Marklund, (1974), GST (glutathione-S-transferase) by Habig et al. (1974), GR (glutathione reductase) by Carlberg and Mannervik, (1985), GPx (glutathione peroxidase) by Hafeman et al. (1984), LPO (Lipid peroxidation) by Stocks and Dormandy, (1971). Differentially modified levels of all biochemical parameters in control rats and rats collected from Bathinda region were assessed for their correlation with oxidative stress.

Histological studies: For histomorphological studies, liver and kidney tissues of the rats collected from Bathinda region and PAU, Ludhiana were placed in alcoholic Bouin's fixative for $24 \mathrm{~h}$ and then dehydrated in graded series of alcohols, cleared in benzene and embedded in paraffin wax (melting point $58-60^{\circ} \mathrm{C}$ ). The $5 \mu \mathrm{m}$ thick sections were cut serially using microtome and stained with haematoxylin and eosin by routine procedures and slides were observed under
OLYMPUS CH20i microscope and photographed.

Statistical analysis: All statistical comparisons for organ weight and biochemical analysis were presented as the mean \pm standard error of mean (S.E.M). Comparisons were made between control and Bathinda rats belonging to different species on computer using t-test. A "P" value of 0.05 was selected as a criterion for statistically significant differences.

\section{RESULTS AND DISCUSSION}

Organ weight: The relative liver and kidney weight decreased slightly in all Bandicota bengalensis rats $(5.02 \pm 0.06$ to $5.00 \pm 0.09$ in liver; $0.41 \pm 0.02$ to $0.4 \pm 0.00$ in kidney) and Tatera indica rats $(4.28 \pm 0.12$ to $4.14 \pm 0.05$ in liver; $0.33 \pm 0.01$ to $0.31 \pm 0.01$ in kidney) collected from Bathinda region of south-west Punjab when compared with the control rats (Fig. 1). Non -significant changes in organs weight observed in present study were comparable with literature where pesticides intoxication does not alter organs weight significantly in rats (Sharma and Sangha 2014).

Biochemical observations: Total protein content was found to be non-significantly lower in the liver (Table 1) and kidney (Table 2) of female $B$. bengalensis as well as liver of Tatera indica collected from Bathinda region while significant decrease in concentration of proteins $(3.80 \pm 0.07$ to $3.62 \pm 0.03)$ was observed in kidney of $T$. indica collected from Bathinda district when compared to the control rats of same species (Table 1). The activity of catalase and glutathione peroxidase was slightly reduced in liver and kidney of female $B$. bengalensis and $T$. indica collected from Bathinda region as compared to the control rats. GST activities were also lowered in liver and kidney of both the species of rats of study area. SOD activity was enhanced significantly in liver $(11.36 \pm 0.91$ to $12.36 \pm 1.10$ ) of $T$. indica collected from Bathinda region as compared to the control rats. The activity of GR increased non-significantly in kidney of $B$. bengalensis and $T$. indica collected from Bathinda

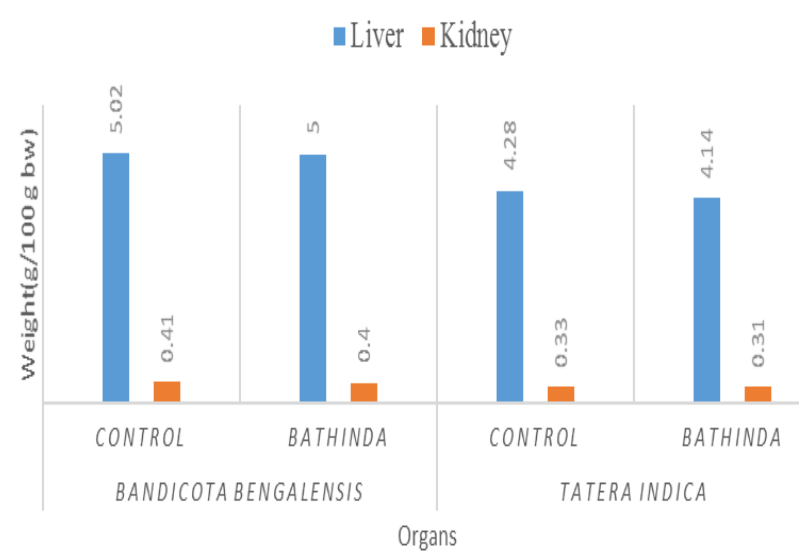

Fig. 1. Relative weight of vital organs $(g / 100 \mathrm{~g} \mathrm{bw})$ in different species of female rats. 


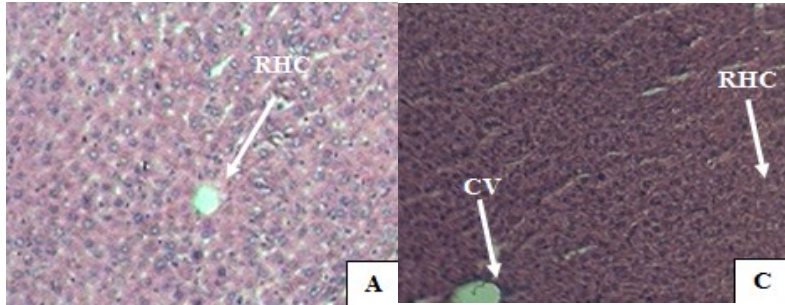

Plate 1: Fig. A and C. T.S. of liver of a control female Bandicota bengalensis (Fig A) and Tatera indica (Fig C) showing normal outlines of the central vein $(\mathrm{CV})$, radially arranged hepatic cords (RHC). Hepatocytes were organized into plates separated by vascular channels (sinusoids) (X 100).

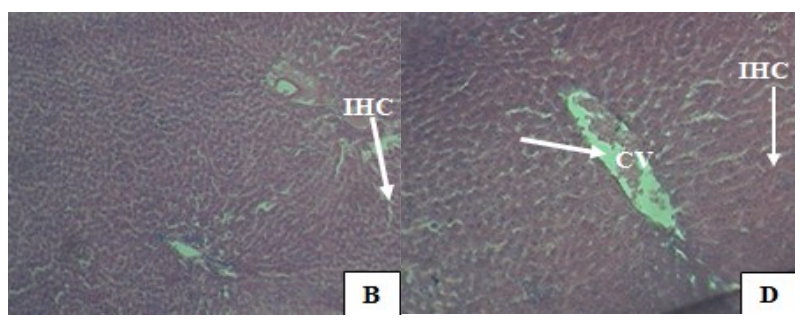

Fig. B and D. T.S. of liver of a female Bandicota bengalensis (Fig B) and female Tatera indica (Fig D) collected from Bathinda district showing slight loosening in arrangement of hepatic cords (IHC), dilation of $\mathrm{CV}$ in the liver tissue and infiltration of leucocytic infilammatory cells (X100).

district when compared to the control rats (Table 2). Significant increase in LPO levels, in terms of MDA, were observed in liver and kidney of all the rats collected from Bathinda region as compared to control rats (Table 1-2).

Under normal physiological conditions, a delicate balance exists between the rate of formation of $\mathrm{H}_{2} \mathrm{O}_{2}$ via dismutation of $\mathrm{O}_{2}$ - by SOD activity and the rate of removal of $\mathrm{H}_{2} \mathrm{O}_{2}$ by CAT and GPx enzymes in the cascade. GST is the major phase II detoxification enzyme and play important role in metabolism of xenobiotic substances. GST activity was non-significant in all rats of study area in comparison to control rats. Similar non-significant observations have been reported for GST activities of ethion and monocroptophos treated rats as compared to control. The glutathionedependent antioxidant system consists of glutathione and two enzymes: GPx and GR (Sharma and Sangha 2014). The biological function of GPx is to reduce lipid hydroperoxides conversion to their corresponding alcohols and to reduce free hydrogen peroxide reaction. Subchronic exposure of rats to dimethoate also caused a significant increase in the activity of GPx in erythrocytes. Malondialdehyde (MDA) is an end product of lipid peroxidation including phospholipids in the cell membrane and the enhanced levels of MDA is an indicator of oxidative stress (Sharma and Sangha 2014). The decreased activity of CAT seen in the poisoning cases coupled with an

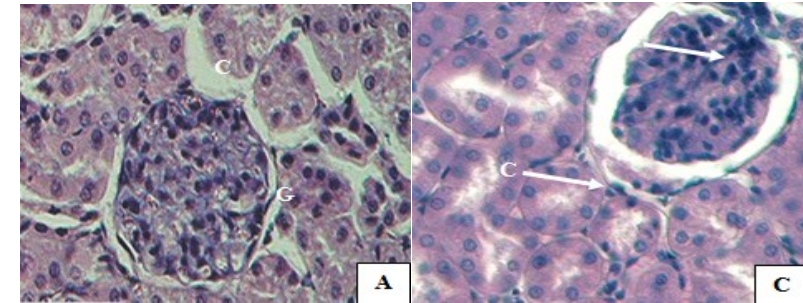

Plate 2: Fig. A and C. Photomicrograph of a T.S. of kidney of a control B. bengalensis(Fig. A) and T. indica (Fig. C) female rat showing intact capsule with well demarcated glomerular tuft $(G)$ and normal arrangement of tubules in the cortical portions $(C)(X 400)$.

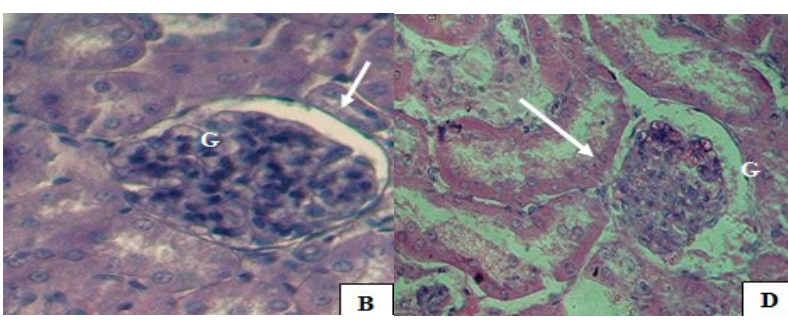

Fig. B. Light micrograph of a Fig. D. Light micrograph of a T.S. of kidney of B. ben- T.S. of kidney of T.indica galensis female rat collected female rat collected from from Bathinda region show Bathinda region showing from Bathinda region show- Bathinda region showing ing enlargement in parietal swelling with mild inflammalayers of Bowman's capsule tory cell infiltration (arrow) (arrow) (X400). (X400)

increase in the lipid peroxidation level (MDA) suggests an insufficient antioxidant defense which could be due to both increase in pesticide-induced ROS formation. The increased levels of MDA along with reduced SOD activity may pose the survival threat to live cells, which may have the potential to affect various organs and their normal physiology leading to severe pathophysiological conditions.

Histological studies in liver: Mammalian liver, by virtue of its unique relationship with the gastrointestinal tract and its role in xenobiotic metabolism, is a target organ of xenobiotic stress. Disturbed liver homeostasis under such stress is sufficient to alter normal body physiology of any organism. Liver is a hub for protein synthesis, regulating cell functions such as maintenance of cellular rigidity, flow management of material through cell membranes, catalysis of an extraordinary range of chemical reactions, regulation of metabolic concentration, and arrangement of nuclear material to control gene function (Bhushan et al., 2013).

Histological observations were recorded in liver of all the rats collected from PAU and Bathinda region. Light microscopic observation of the liver sections of the control rats showed a normal histological architecture of liver of female rats clearly outlined in the sections of anastomosing hepatocytes along with adjacent sinusoids radiating from the central veins towards the periphery of the liver lobules (Plate 1, Fig 
Table 1. Liver enzymatic antioxidant parameters ( $\mathrm{mg} \mathrm{g}^{-1}$ wet weight of tissue) of Bandicota bengalensis and Tatera indica female rats.

\begin{tabular}{|c|c|c|c|c|}
\hline \multirow{2}{*}{ Parameters } & \multicolumn{2}{|c|}{ Bandicota bengalensis } & \multicolumn{2}{|c|}{ Tatera indica } \\
\hline & Control & Bathinda & Control & Bathinda \\
\hline Protein $(\mathrm{mg} / \mathrm{g}$ tissue $)$ & $4.22 \pm 0.28$ & $4.21+0.24$ & $4.12 \pm 0.32$ & $3.97+0.37$ \\
\hline CAT ( $\mu$ mole of $\mathrm{H}_{2} \mathrm{O}_{2}$ decomposed $/ \mathrm{min} / \mathrm{mg}$ protein) & $45.66 \pm 3.24$ & $43.70 \pm 7.55$ & $53.02 \pm 8.76$ & $49.39 \pm 7.81$ \\
\hline $\mathrm{SOD}(\mathrm{U} / \mathrm{mg}$ protein $)$ & $11.52 \pm 0.58$ & $11.76 \pm 1.83$ & $11.36 \pm 0.91$ & $12.36 \pm 1.10^{*}$ \\
\hline GST ( $\mu$ moles of GSH-CDNB conjugate formed $/ \mathrm{min} / \mathrm{mg}$ protein) & $0.41 \pm 0.02$ & $0.41 \pm .02$ & $0.40 \pm 0.007$ & $0.40 \pm 0.06$ \\
\hline GR ( $\mu$ moles of NADPH conjugate/ $\mathrm{min} / \mathrm{mg}$ protein $)$ & $0.07 \pm 0.01$ & $0.07 \pm 0.01$ & $0.06 \pm 0.02$ & $0.07 \pm 0.01$ \\
\hline GPx (U/mg protein) & $0.32 \pm 0.01$ & $0.28 \pm 0.06$ & $0.34 \pm 0.11$ & $0.32 \pm 0.13$ \\
\hline LPO (nM MDA/100 mg tissue) & $11.05 \pm 0.18$ & $11.60 \pm 0.10$ & $9.02 \pm 0.26$ & $9.43 \pm 2.58$ \\
\hline
\end{tabular}

All the values are Mean \pm SE of 5 animals in each group, *Statistically significant different $(p \leq 0.05)$ as compared to control

Table 2. Kidney enzymatic antioxidant parameters ( $\mathrm{mg} \mathrm{g}^{-1}$ wet weight of tissue) of Bandicota bengalensis and Tatera indica female rats.

\begin{tabular}{|c|c|c|c|c|}
\hline \multirow{2}{*}{ Parameters } & \multicolumn{2}{|c|}{ Bandicota bengalensis } & \multicolumn{2}{|c|}{ Tatera indica } \\
\hline & Control & Bathinda & Control & Bathinda \\
\hline Protein (mg/g tissue) & $4.79+0.01$ & $4.70+0.40$ & $3.80 \pm 0.07$ & $3.62+0.30^{*}$ \\
\hline CAT ( $\mu$ mole of $\mathrm{H}_{2} \mathrm{O}_{2}$ decomposed $/ \mathrm{min} / \mathrm{mg}$ protein) & $45.80 \pm 2.84$ & $45.78 \pm 4.42$ & $45.40 \pm 1.01$ & $42.76 \pm 3.59$ \\
\hline SOD (U/mg protein) & $12.02 \pm 0.38$ & $12.00 \pm 1.66$ & $12.00 \pm 0.51$ & $12.17 \pm 1.37 *$ \\
\hline GST ( $\mu$ moles of GSH-CDNB conjugate formed $/ \mathrm{min} / \mathrm{mg}$ protein) & $0.47 \pm 0.01$ & $0.45 \pm 0.06$ & $0.42 \pm 0.03$ & $0.41 \pm 0.03$ \\
\hline GR ( $\mu$ moles of NADPH conjugate/ $\mathrm{min} / \mathrm{mg}$ protein $)$ & $0.06 \pm 0.01$ & $0.076 \pm 0.02$ & $0.067 \pm 0.01$ & $0.069 \pm 0.01$ \\
\hline GPx (U/mg protein) & $0.37 \pm 0.02$ & $0.33 \pm 0.13$ & $0.37 \pm 0.02$ & $0.35 \pm 0.04$ \\
\hline LPO (nM MDA/100 mg tissue) & $10.03 \pm 0.44$ & $10.18 \pm 0.72$ & $8.68 \pm 0.24$ & $8.92 \pm 4.34$ \\
\hline
\end{tabular}

All the values are Mean \pm SE of 5 animals in each group, *Statistically significant different $(\mathrm{p} \leq 0.05)$ as compared to control

A,C). Normal outlines of the central vein (CV) can be clearly visualized as shown. Hepatocytes nuclei are round with dispersed chromatin and prominent nucleoli. Hepatocytes were organized into plates separated by vascular channels (sinusoids). No infiltration of leucocytes was observed in the sinusoids . Each lobule was bounded by scanty connective tissue (Plate 1, Fig. A,C).

The obtained results were found to be supported by the results of Owoeye et al. (2012) who reported that the liver of control rats showed a normal structure with hexagonal lobules, central veins and peripheral triads embedded in connective tissue. The hepatocytes are arranged in trabecules running radially from the central vein and are separated by sinusoids, and each of the hepatocytes contained a large spheroidal nucleus. The results also coincides with Hassar and Mazzan (2010) who observed that the hepatic parenchyma of the control rats consisted of several hepatic lobules separated from each other by very delicate connective tissue septa housing the portal triad. Each hepatic lobule contained a thin walled central vein surrounded by hepatic cords radiating towards the periphery.

Liver of Bathinda female rats showed slight loosening in arrangement of hepatic cords around central vein (Plate 1, Fig. B). Large vacuolization or empty spaces in all the rats were observed to be prominent be maximum in $B$. bengalensis. The dialation of $\mathrm{CV}$ and sinusoids between hepatocytes were also increased (Plate 1, Fig. B, D) and showed infiltration of large mass of leucocytes inflammatory cells in CV and sinusoids.

Hassar and Mazzan (2010) also observed generalized dilation and congestion involving the hepatic arteries, lymph vessels, central veins and hepatic sinusoids. The hepatocytes appeared vacuolated ballooned and some of them showed degenerative changes while the nuclei appeared pyknotic and densely stained in rats. Histopathological examination of the liver from animals treated with cypermethrin and beta-cyfluthrin also revealed various cellular and lobular abnormalities, including intralobular vein (ILV) membrane dilation, presence of hepatocytes in ILV, cytoplasmic vacuolisation, multinuclear cells, nuclear polymorphisms, nuclear vacuolisation, hepatocyte membrane damage, nuclear division, nuclear eccentricity, pyknosis, necrosis, and karyorrhexis (Bhushan et al., 2013). Heikal et al. (2013) reported histopathological alterations in liver of cyromazine and chlorpyrifos treated rats which include degeneration and coagulative necrosis in the hepatocytes, inflammatory cells infiltration, and kupffer cells proliferation.

Histological studies in kidney: Histopathological examination photomicrographs of the kidney sections in the control female $B$. bengalensis and $T$. indica showed a well demarcated medulla and cortex with intact capsule and well-formed glomerular tuft (Plate2, Fig. A, C). It reflects the normal integrity of the cortex and blood vessels.

Kidney sections of female rats collected from Bathinda district showed variable changes in some parts of the urinary tubules and distension of glomerular capillaries in $B$. bengalensis (Plate-2, Fig. B) and T. indica 
(Plate-2, Fig. D). Sections of kidney of B. bengalensis (Plate-2, Fig. B) and T. indica (Plate-2, Fig. D) showed swelling appearances, increasing urinary space, inflammatory cell infiltration degeneration of glomaruli and associated tubules structure.

The histological structure of kidney tissue of rats in the control group, and rats exposed to endosulfan showed that the renal cortex area showed more pronounced histopathological changes in endosulfan treated animals, compared with the control (Khan and Kumari 2011). Histology of renal tissue obtained for the control rats showed normal renal corpuscles, consisting of a tuft of capillaries (Khan and Kumari 2011). The kidneys of the rats administrated profenofos at three doses level showed histopathological lesions which are represented by heamorrhages, periglome- rular edema, and some glomeruli were shrinkage (Hammam and ElMottaleb 2007). Also, necrosis of some cells lining the renal tubules was observed. In $1 / 80 \quad \mathrm{LD}_{50}$ of profenofos administrated rats, kidneys showed formation of renal cast in the lumen of the renal tubules (Hammam and El-Mottaleb, 2007).

\section{Conclusion}

In the present study, examination of liver and kidney functions were correlated with the histopathological changes. The observations indicated marked changes in the overall histo architecture of these organs which could be due to pesticides toxic effects primarily by the generation of ROS, causing damage to the various membrane components of the cell. Differentially altered activity levels of number of enzymes and increased level of LPO was observed in present study which is in corroboration to the severe histopathological damages due to pesticide exposure. However, further studies are needed to explore the molecular mechanism, underlying the role of pesticides to induce oxidative stress.

\section{REFERENCES}

Abdollahi, M., Ranjbar, A., Shadnia, S., Nikfar, S. and Rezaiee, A. (2004). Pesticides and oxidative stress: a review. Medical Sci. Monitor, 10(6): 141-147

Aebi, H. (1983). Catalase In: Bergmeyer H U and Weinheim (ed) Methods of Enzymatic Analysis. Pp. 227-82. Academic Press.

Bhushan, B., Saxena, P. N. and Saxena, N. (2013). Biochemical and histological changes in rat liver caused by cypermethrin and beta-cyfluthrin. Arh. Hig. Rada Toksikol., 64: 57-67

Carlberg, I. and Mannervik, B. (1985). Glutathione reductase. Methods Enzymol., 113:484-90

Chattopadhyay, S., Podder, S., Agarwal, S. and Bhattacharya, S. (2011). Fluoride-induced histopathology and synthesis of stress protein in liver and kidney of mice. Ach. Toxicol., 85: 327-35

Habig, W. H., Pabst, M. J. and Jakoby, W. B. (1974). Glutathione S-transferases. The first enzymatic step in mer- capturic acid formation. J. Biol. Chem., 246:7130-7139

Hafeman, D. G., Sunde, R. A. and Hoekstra, W. G. (1984). Effect of dietary selenium erythrocyte and liver glutathione peroxidise in the rat. J. Nutr., 104:580-587

Hashmi, I. and Khan, A. D. (2011). Adverse health effects of pesticides exposure in agricultural and industrial workers of developing country. Pesticides-The Impacts of Pesticides Exposure. http://dx. doi. org/10.5772/ 13835.

Hammam, M. A. and Abdel-Mottaleb, E. M. (2007). Studies of the genotoxic and histopathological effects of the organophosphorus insecticide "profenofos" on white rats. Egypt J. Hosp. Med., 29: 685-706

Hassar, G. M. and Mazzan, K. H. M. (2011). Genotoxicity and histopathological studies on the liver, kidney and lymphocytes of male rats fed on diet containing waste fat released from chicken during grilling process. $J$. Cytol. Histol., 2:111

Heikal, T., Mossa, A. T., Rasoul, M. and Marei, H. (2013). The ameliorating effects of green tea extract against cyromazine and chlorpyrifos induced liver toxicity in male rats. Asian J. Pharma. Clin. Res., 6(1):48-55

Khan, S. and Kumari, D. O. O. J. (2011). Analytical study of histopathological changes induced by of Endosulfan in kidney of albino rats. Asian J. Pharma.Clin. Res., 5(1): $113-114$

Lowry, O. H., Rosebrough, N. J., Farr, A. L. and Randall, A. J. (1951). Protein measurement with folin phenol reagent. J. Biol. Chem., 193:265-275

Marklund, S. and Marklund, G. (1974). Involvement of the superoxide anion radical in the autoxidation of pyrogallol and a convenient assay for superoxide dismutase. Euro. J. Biochem., 47:469-474

Mansour, S.A and Mossa, A.H. (2010). Oxidative damage, biochemical and histopathological alterations in rats exposed to chlorpyrifos and the antioxidant role of zinc. Pesticide. Biochem. Physiol., 96: 14-23. DOI: 10.1016/ j.pestbp.2009.08.008.

Maroni, M., Colosio, C., Ferioli, A. and Fait, A. (2000). Toxicology: review. Toxicology, 143; 5-91

Mittal, S., Kaur, G. and Vishwakarma, G. S. (2014). Effects of Environmental Pesticides on the Health of Rural Community in the Malwa Region of Punjab, India: A Review". Human Ecol. Risk Assessment Int. J., 20 (2): 366-387

Owoeye, O., Edem, F., Akinyoola, B., Rahaman, S., Akang, E. and Arinola, G. (2012). Histological changes in liver and lungs of rats exposed to dichlorvos before and after vitamin supplementation. Eur. J. Anat., 16 (3): 190-198

Sharma, D. and Sangha, G. K. (2014). Triazophos induced oxidative stress and histomorphological changes in liver and kidney of female albino rats. Pesticide Biochem. Physiol., 110: 71-80

Shetty, P. K. (2004). Socioecological implications of pesticide use in India. Economic and Political Weekly, 5261 $-5267$

Stocks, J. and Dormandy, T. L. (1971). The autoxidation of human red cell lipids induced by hydrogen peroxide. British J. Haem., 20(1):95-111

Thakur, J. S., Rao, T. B., Rajwanshi, A., Parwana, H. K. and Kumar, R. (2008). Epidemilogical study of high cancer among rural agricultural community of Punjab in northern India. Int. J. Environ. Res. Pub. Health, 5:399-4 